\title{
Status of the Problem of Nuclear Cross Section Data for IBA
}

\author{
A. Gurbich ${ }^{* 1}$, I. Bogdanovic-Radovic ${ }^{2}$, M. Chiari ${ }^{3}$, C. Jeynes ${ }^{4}$, M. Kokkoris ${ }^{5}$, \\ A.R. $\operatorname{Ramos}^{6}$, M. Mayer ${ }^{7}$, E. Rauhala ${ }^{8}$, O. Schwerer ${ }^{9}$, Shi Liqun ${ }^{10}$, I. Vickridge ${ }^{11}$ \\ ${ }^{1}$ Institute of Physics and Power Engineering, Bondarenko 1, 249033, Obninsk, Russia \\ ${ }^{2}$ Ruder Bošković Institute, Bijenička c. 54, P.O. Box 180, 100002, Zagreb, Croatia \\ ${ }^{3}$ Insituto Nazionale Fisica Nucleare, Via Sansone 1, Sesto Fiorentino, I-50019, Firenze, Italy \\ ${ }^{4}$ University of Surrey Ion Beam Centre, Guildford GU2 7XH, England \\ ${ }^{5}$ National Technical University of Athens, Zografou Campus, 15780 Athens, Zografou, Greece \\ ${ }^{6}$ Instituto Tecnológico e Nuclear, Estrada Nacional 10, 2686-953 Sacavém, Portugal \\ ${ }^{7}$ Max-Planck-Institut für Plasmaphysik, EURATOM Association, Boltzmannstr. 2, D-85748 Garching, \\ Germany \\ ${ }^{8}$ University of Helsinki, Accelerator Laboratory, P.O. Box 64, 00014 Helsinki, Finland \\ ${ }^{9}$ International Atomic Energy Agency, Wagramer Strasse 5, A-1400 Vienna, Austria \\ ${ }^{10}$ Fudan University, Institute of Modern Physics, Shanghai 200433, China \\ ${ }^{11}$ Institut des NanoSciences de Paris, UMR7588 du CNRS, Université de Pièrre et Marie Curie, Paris, France
}

* Corresponding author:

A. Gurbich

Institute of Physics and Power Engineering,

Bondarenko sq. 1,

Obninsk, 249032,

Russian Federation

Phone: +7 4843994169

Fax: +7 4843958477

E-mail: gurbich@ippe.ru 


\begin{abstract}
The progress achieved in an IAEA Coordinated Research Project (CRP) to improve the cross section data for IBA is reported. The objective of the CRP, started in 2005, is to create a nuclear cross section database for IBA that contains reliable and usable data freely available to the entire IBA community. The major results achieved so far by the CRP participants are discussed. The results include compilation and assessment of the existing cross sections, new experimental data, and evaluation of the most wanted cross sections. The experimental results are incorporated into the IBANDL database (www-nds.iaea.org/ibandl/) and evaluated data are presented at the SigmaCalc cross section calculator (wwwnds.iaea.org/sigmacalc/).
\end{abstract}

Key words: elastic scattering; nuclear reaction; cross section; measurement; assessment; evaluation

PACS: 25.60.Bx; 25.45.-z; 29.87.+g 


\section{Introduction}

The lack of reliable cross sections was recognized by the IBA community long ago [1] and was discussed in several workshops at biannual IBA conferences and also at International Atomic Energy Agency (IAEA) meetings [2-3]. As a way to resolve the problem the Coordinated Research Project (CRP) "Development of a Reference Database for Ion Beam Analysis" was initiated by the IAEA after consultation with the ion beam analysis community. The ultimate goal of the CRP is to produce a nuclear reaction cross section database containing recommended data of relevance to the IBA. The actions to reach this goal are compilation of the information scattered in the literature, critical assessment of the compiled data, the performance of new measurements when there are no data available or where unresolved discrepancies exist, evaluation of the cross sections, incorporation of all measured and evaluated data into the database, and making them available to the IBA community.

The need for recommended cross sections arises from the fact that cross section data used in IBA are not only sparse and insufficient but they are often inconsistent. An established procedure for the elaboration of the recommended cross section does not exist. Actually, evaluated cross sections are usually regarded as recommended. However, to evaluate all the data of interest for IBA is beyond the capability of this CRP. So the most wanted data will be evaluated, and recommendations for the rest of the data will be based on assessments and on results of benchmark experiments.

While planning the scope of the CRP activity it was decided that a strong emphasis should first be given to elastic scattering of protons and alphas from light elements, since these cross sections are widely used, and nuclear theory exists enabling valid evaluations to be made. A secondary emphasis is placed on deuteron-induced nuclear reactions, which often have advantages of high selectivity and sensitivity in the determination of light elements. 
The aim of the present paper is to present the methodology employed and the results achieved so far in implementation of the CRP.

\section{Compilation}

Following recommendations of the IAEA Technical Meeting [3] a new database IBANDL (Ion Beam Analysis Nuclear Data Library) was established at the IAEA Nuclear Data Section (NDS) server (www-nds.iaea.org/ibandl/). Its official start was announced in April 2004. To begin with, the data from SigmaBase (still available at www.mfa.kfki.hu/sigmabase/) and NRABASE [4] were combined within an advanced design. Since then the number of files in IBANDL has increased from 440 to 876 . Now IBANDL contains most (but still not all!) of the available experimental nuclear cross sections relevant to IBA and much of the data on PIGE (particle induced gamma emission: including DIGE - deuteron IGE).

IBANDL is being filled both with results of new measurements and with cross sections found in the literature. The data published only in a graphical form are digitized using a precise technique. Their reliability was verified by the comparison of the digitized data with tabulated ones for several cases when data were presented in the literature both in graphical and tabular forms. It was proved that the technique was accurate within 2-5 per cent depending on the quality of the published figure. Thus the additional uncertainty caused by digitizing has the same value as the usual experimental uncertainty. The current content of IBANDL is shown in Figs. 1-2. In order to encourage members of the IBA community to share their cross section data there is a template at the IBANDL site, which makes it easy to upload new data files even for inexperienced users.

Another source of the cross section data potentially useful for IBA is the EXFOR database (www-nds.iaea.org/exfor/). While this database has traditionally been the main source of experimental neutron cross section data, its completeness for charged particle data 
has improved considerably only in the past years, and in particular, many differential data for IBA have been included.. Thanks to activity of the NDS staff (S. Dunaeva) the number of the IBA relevant data in EXFOR grows very fast. The EXFOR format is used for many more data types than needed in IBANDL, and was developed to be extremely versatile, therefore it is more complicated. All the IBANDL data are stored in the R33 format [5]. The ASCII R33 format was proposed in 1991 for the communication and accumulation of IBA cross section data and was several times updated since then. A current version of the format description is available at www-nds.iaea.org/ibandl/r33.html. A special R33 manager was developed by one of the authors (I.V.) and some codes used in IBA for spectra processing were adapted to this format. The R33 format was developed $a b$ ovo with no relation to EXFOR. There are several principal differences between EXFOR and IBANDL. Data are compiled in EXFOR as they are published whereas angles and cross sections in IBANDL are always presented in the laboratory frame of reference. Excited nucleus states are referred by numbers in IBANDL and by excitation energies in EXFOR. Besides the R33 files include some information not normally included in EXFOR (e.g., reaction Q values); on the other hand, EXFOR contains much additional information (such as on the measurement method and other textual information) which is not included in IBANDL. The comparison between IBANDL and EXFOR revealed some differences in data files corresponding to the same publication (e.g., slight discrepancies in the numerical values, different number of angles in the data files, etc.). The work is currently under way to synchronize the EXFOR and IBANDL databases including the development of an additional output R33 format for EXFOR database (V. Zerkin, NDS).

The analysis of IBANDL site statistics revealed the most popular 15 cross sections, which cover more than $90 \%$ of retrievals (see Fig. 3). It is evident that elaboration of the recommended cross sections for these cases is of primary importance. 


\section{Assessment}

By assessment it is intended here the scrutinizing of the available information for a particular cross section, with the aim of drawing conclusions as to its reliability and accuracy. In particular, completeness of the data contained in IBANDL is verified through thorough literature search. Mistakes found in the compiled cross sections are corrected and gaps in energy and angle intervals are identified. Inconsistencies in the available experimental data are revealed and analyzed based on the critical study of experimental details. Possible sources of unaccounted errors are looked for. Cases for which new measurements are needed are pointed out.

Assessment was found to be a very useful and productive procedure that should precede any further work with nuclear data. So all the cross sections potentially useful for IBA were assigned to the CRP participants for assessment. The obtained results will be used as a basis for elaboration of the recommended cross sections.

\section{Measurements}

The cross section measurements planned in the framework of the CRP are summarized in Table 1. The elements for the measurements were selected on the basis of the analysis of the previously acquired data, taking account of the availability of targets. The great variety of facilities at the disposal of different CRP participating groups make it possible to cover a wide interval of angles and a vast energy range. Every effort is made in order to avoid mistakes and pitfalls in the cross section measurements. The problems of accelerator energy calibration, target preparation and characterization, peak area determination, and estimation of the uncertainty budget were discussed at the CRP meetings. To avoid errors of charge integration and solid angle determination internal standards are used as far as possible. Measured cross sections are uploaded onto IBANDL. 
In addition to the cross section measurements benchmark experiments consisting of measuring charged particle spectra from well characterized uniform thick targets are also planned within the CRP. Whilst it should, in principle, be possible to derive cross sections from such experiments, it was reported that even using advanced inversion techniques, the derived cross sections were inferior to those obtained from thin targets. Nevertheless, thick target benchmark experiments provide a convincing test of the overall validity for IBA of the measured cross sections. Such validation depends on the availability of tested simulation codes and valid stopping powers. Within this CRP, the SIMNRA [6] or Data Furnace [7] simulation codes and SRIM-2003 [8] stopping powers are adopted to simulate particle spectra that correspond to a given cross section. An example demonstrating the power of benchmarking in resolving inconsistencies in the available data can be found in [9].

\section{Evaluation}

Nuclear data evaluation is a routine procedure in preparing neutron data for different applications. The same approach was successfully applied to the charged particle cross sections (see [10] and references therein). Theoretical aspects of the charged particle cross section evaluation can be found elsewhere [11]. The evaluation starts with the assessment followed by theoretical calculations of the cross section in the framework of the appropriate nuclear physics model. Free parameters of the model are adjusted to fit all available data. Thus differential cross sections measured under different angles are incorporated in the analysis within a unified approach. Furthermore, information additional to direct measurement of the cross section under consideration (such as adopted resonance parameters, cross sections for different reaction channels, and total cross sections) can be included in the analysis. All this makes the evaluated cross sections more reliable than the results of any particular measurement. When the cross section has been evaluated it can be calculated for any scattering angle. The calculations are implemented remotely at the SigmaCalc site 
(www-nds.iaea.org/sigmacalc/). Being rather complicated these are arranged in such a way that demands no expertise in nuclear physics from a potential user. The changes made recently in the IBANDL design enable a user to make calculations directly from the IBANDL page presenting experimental data for a particular nucleus.

The evaluated cross sections are listed in Table 2. As is seen from the comparison of the content of Table 2 with the IBANDL retrieval statistics presented in Fig. 3 the most wanted cross sections have been already evaluated except for ${ }^{14} \mathrm{~N}(\alpha, \alpha){ }^{14} \mathrm{~N},{ }^{19} \mathrm{~F}(\mathrm{p}, \mathrm{p}){ }^{19} \mathrm{~F}$, and deuteron induced reactions. The work on evaluation is in progress including development of the necessary computer codes for deuteron induced reactions.

\section{Conclusion}

Coordinated effort is being applied by the CRP participants to resolve the most urgent problems in nuclear data for IBA. The work will be finished approximately in year 2008 by preparing recommended cross sections. The recommended data will be put into IBANDL where they can be easily compared with available experimental data using recently designed overlay graphics.

\section{Acknowledgements}

This work was done as a part of the IAEA Coordinated Research Project "Development of a Reference Database for Ion Beam Analysis". One of the authors (A.G.) wishes to acknowledge the support by Sandia National Laboratories through the ISTC project 3748p. 


\section{References}

1. I.C. Vickridge, Nucl. Instr. and Meth. B66 (1992) 303.

2. Summary Report of the Advisory Group Meeting on Long-Term Needs for Nuclear Data Development, Vienna, 28 November - 1 December 2000, INDC(NDS)-423, Vienna, 2001.

3. Report of IAEA Technical Meeting on Database of Evaluated Cross Sections for Ion Beam Applications, Vienna, 29 - 30 October 2003, INDC(NDS)-449, Vienna, 2003.

4. A.F. Gurbich, A.V. Ignatyuk, in: G. Reffo, A. Ventura, C. Grandy (Eds.), Nuclear Data for Science and Technology, Conf. Proc., vol. 59, SIF, Bologna, 1997, p. 1740.

5. I.C. Vickridge, DSIR Physical Sciences Report 33, Lower Hutt, New Zealand, 1991.

6. M. Mayer, Technical Report IPP9/113, Max-Planck-Institut für Plasmaphysik, Garching, Germany (1997); . Mayer, AIP Conference Proceedings 475 (1999) 54; M. Mayer, Nucl. Instr. and Meth. B194 (2002) 177

7. N.P. Barradas, C. Jeynes, and R.P. Webb, Appl. Phys. Lett. 71 (1997) 291; N.P. Barradas, P.K. Marriott, C. Jeynes, R.P. Webb, Nucl. Instr. and Meth. B136-138 (1998) 1157; C. Jeynes, N.P. Barradas, P.K. Marriott, G. Boudreault, M. Jenkin, E. Wendler and R.P. Webb, J. Phys. D: Appl. Phys. 36 (2003) R97

8. www.SRIM.org; J.F. Ziegler, Nucl. Instr. and Meth. B219-220 (2004) 1027. The current version (SRIM2006) has stopping powers indistinguishable from SRIM2003

9. A. Gurbich, C. Jeynes, Nucl. Instr. and Meth. in press.

10. A.F. Gurbich, in: R.C. Haight, M.B. Chadwick, T. Kawano, P. Talou (Eds.), Nuclear Data for Science and Technology, AIP Conf. Proc., Vol. 769, Melville, New York, 2005, p. 1670.

11. A.F. Gurbich, Nucl. Instr. and Meth. B261 (2007) 401. 
Table 1. Cross section measurements to be made in the CRP framework

\begin{tabular}{|c|c|c|c|c|}
\hline Target & Reaction & $\begin{array}{l}\text { Energy } \\
(\mathrm{MeV})\end{array}$ & $\begin{array}{l}\text { Angular range } \\
\text { (degree) }\end{array}$ & Status \\
\hline \multirow[t]{2}{*}{$\mathrm{D}$} & $(\alpha, \alpha)$ & $3-8$ & $20,30,40$ & In progress \\
\hline & $(\mathrm{p}, \mathrm{p})$ & $0.5-4.0$ & $>135$ & In progress \\
\hline \multirow[t]{2}{*}{$\mathrm{T}$} & $(\alpha, \alpha)$ & $3-8$ & $20,30,40$ & In progress \\
\hline & $(\mathrm{p}, \mathrm{p})$ & $1-3$ & 151,165 & In progress \\
\hline He-3 & $(\mathrm{d}, \mathrm{x})$ & around 0.63 & & In progress \\
\hline $\mathrm{Li}$ & $(p, p)$ & $0.5-6.0$ & $100-170$ & $\begin{array}{l}\text { Measurements } \\
\text { at } E=3-6 \mathrm{MeV} \\
\text { to be started }\end{array}$ \\
\hline Li-6 & $(d, p),(d, \alpha)$ & $0.9-2.0$ & $100-170$ & In progress \\
\hline B-10,11 & $(\mathrm{d}, \mathrm{p}),(\mathrm{d}, \alpha)$ & $0.9-2.0$ & $100-170$ & "Completed \\
\hline C-12 & $(\mathrm{p}, \mathrm{p})$ & $3-6$ & $100-170$ & To be started \\
\hline $\mathrm{C}-13$ & $(\mathrm{~d}, \mathrm{x})$ & & & In progress \\
\hline \multirow[t]{2}{*}{$\mathrm{N}$} & $(\mathrm{p}, \mathrm{p})$ & $2.4-5.0$ & $120-165$ & Completed \\
\hline & $(\alpha, \alpha)$ & $2.0-8.0$ & $120-165$ & In progress \\
\hline $\mathrm{N}-14$ & $(\mathrm{~d}, \mathrm{p}),(\mathrm{d}, \alpha),(\mathrm{d}, \mathrm{d})$ & $0.9-2.0$ & $100-170$ & In progress \\
\hline $\mathrm{N}-15$ & $(\mathrm{~d}, \mathrm{x})$ & & & In progress \\
\hline$\overline{\mathrm{O}}$ & $(\alpha, \alpha)$ & $7-9$ & $100-170$ & In progress \\
\hline \multirow[t]{2}{*}{ F-19 } & $(\mathrm{p}, \mathrm{p})$ & up to 6 & $100-170$ & To be started \\
\hline & $(\mathrm{d}, \mathrm{p}),(\mathrm{d}, \alpha)$ & $0.9-2.0$ & $100-170$ & In progress \\
\hline \multirow[t]{2}{*}{$\overline{\mathrm{Al}}$} & $(\mathrm{d}, \mathrm{p}),(\mathrm{d}, \alpha)$ & $1-2$ & 150 & Completed \\
\hline & $\gamma$-ray yield & $1-2$ & 90 & Completed \\
\hline $\mathrm{Si}$ & $(\alpha, \alpha)$ & $2-8$ & $100-160$ & In progress \\
\hline \multirow[t]{2}{*}{$\mathrm{Ti}$} & $(\alpha, \alpha)$ & $2-6$ & 148,173 & In progress \\
\hline & $(\mathrm{p}, \mathrm{p})$ & $2-4$ & 148,173 & In progress \\
\hline \multirow[t]{2}{*}{$\mathrm{V}$} & $(\alpha, \alpha)$ & $2-6$ & 148,173 & In progress \\
\hline & $(p, p)$ & $2-4$ & 148,173 & In progress \\
\hline
\end{tabular}

Table 2. Evaluated cross sections

\begin{tabular}{|c|c|c|c|c|c|c|c|c|c|c|c|c|c|c|c|}
\hline \multirow{2}{*}{$\begin{array}{l}\text { Eleme } \\
\mathrm{nt}\end{array}$} & \multirow{2}{*}{$\begin{array}{c}{ }^{1} \mathrm{H} \\
(\alpha, p \\
)\end{array}$} & \multirow{2}{*}{$\begin{array}{l}{ }^{4} \mathrm{He} \\
\text { (p,p } \\
\text { ) }\end{array}$} & \multicolumn{2}{|c|}{${ }^{12} \mathrm{C}$} & \multirow{2}{*}{$\begin{array}{c}{ }^{14} \mathrm{~N} \\
(p, p \\
)\end{array}$} & \multicolumn{2}{|c|}{${ }^{16} \mathrm{O}$} & \multirow{2}{*}{$\frac{\begin{array}{c}{ }^{24} \mathrm{M} \\
\mathrm{g}\end{array}}{(\mathrm{p}, \mathrm{p}}$} & \multirow{2}{*}{$\begin{array}{c}{ }^{{ }^{n a t} M} \\
g \\
\text { (p,p } \\
\text { ) }\end{array}$} & \multirow{2}{*}{$\begin{array}{c}{ }^{27} \mathrm{~A} \\
1 \\
(\mathrm{p}, \mathrm{p} \\
)\end{array}$} & \multicolumn{2}{|c|}{${ }^{28} \mathrm{Si}$} & \multicolumn{2}{|c|}{${ }^{\text {nat }} \mathrm{Si}$} & \multirow{2}{*}{$\begin{array}{c}{ }^{32} S \\
(p, p \\
)\end{array}$} \\
\hline & & & $\begin{array}{c}(\mathrm{p}, \mathrm{p} \\
)\end{array}$ & $\begin{array}{l}(\alpha \\
\alpha)\end{array}$ & & $\begin{array}{c}(\mathrm{p}, \mathrm{p} \\
)\end{array}$ & $\begin{array}{l}(\alpha \\
\alpha)\end{array}$ & & & & $\begin{array}{c}(\mathrm{p}, \mathrm{p} \\
)\end{array}$ & $\begin{array}{c}(\alpha, \alpha \\
)^{*}\end{array}$ & $\begin{array}{c}\text { (p,p } \\
\text { ) }\end{array}$ & $\begin{array}{c}(\alpha, \alpha \\
{ }^{*}\end{array}$ & \\
\hline $\begin{array}{l}\text { Max. } \\
\text { energy, } \\
\text { MeV }\end{array}$ & $\begin{array}{c}10 . \\
0\end{array}$ & 2.7 & 3.5 & 7.2 & 3.4 & 4.0 & 4.0 & 2.7 & 2.7 & 1.8 & 3.0 & 4.5 & 3.0 & 4.5 & 3.6 \\
\hline
\end{tabular}

*Not yet available at SigmaCalc 


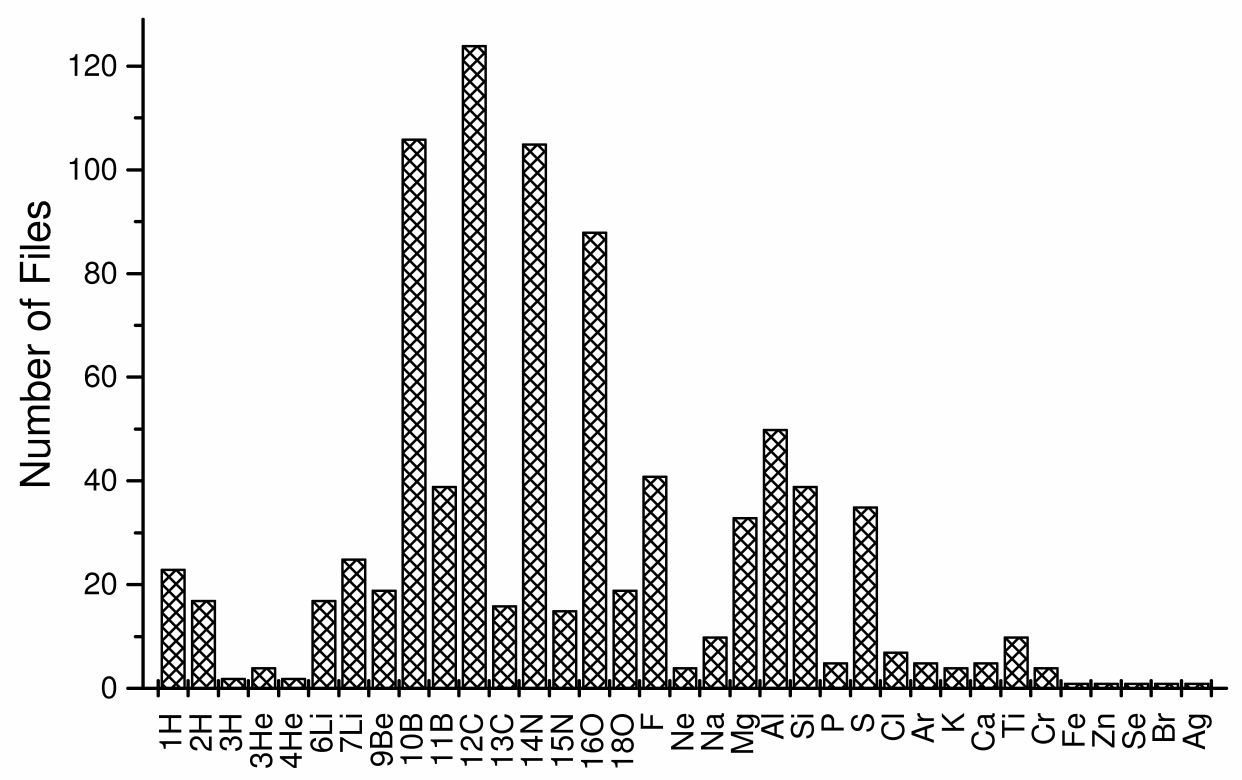

Fig.1. The IBANDL content by target element
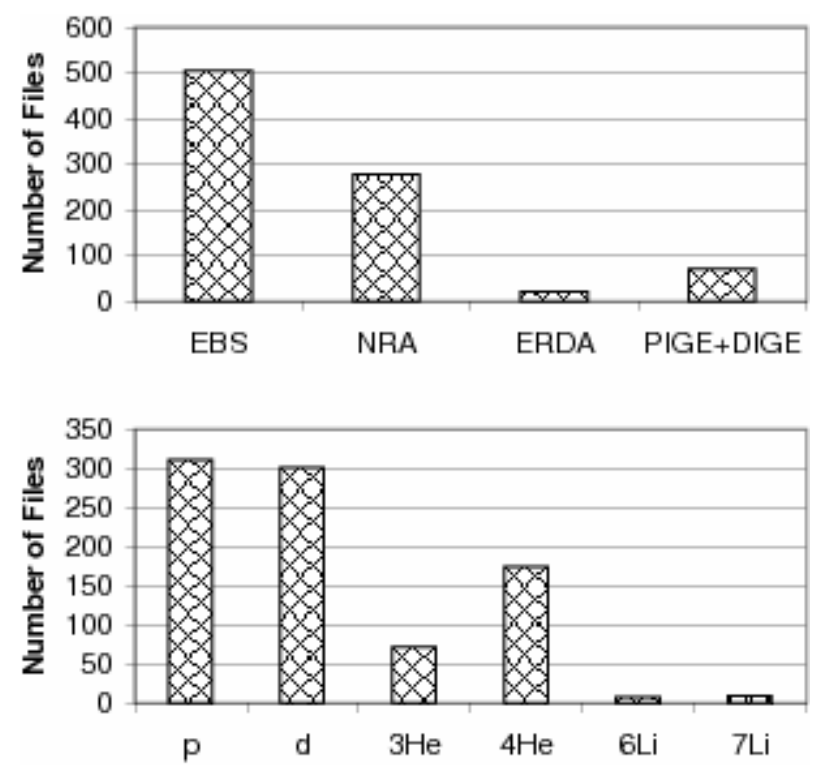

Fig.2. The IBANDL content by reaction (top) and by projectile (bottom) 


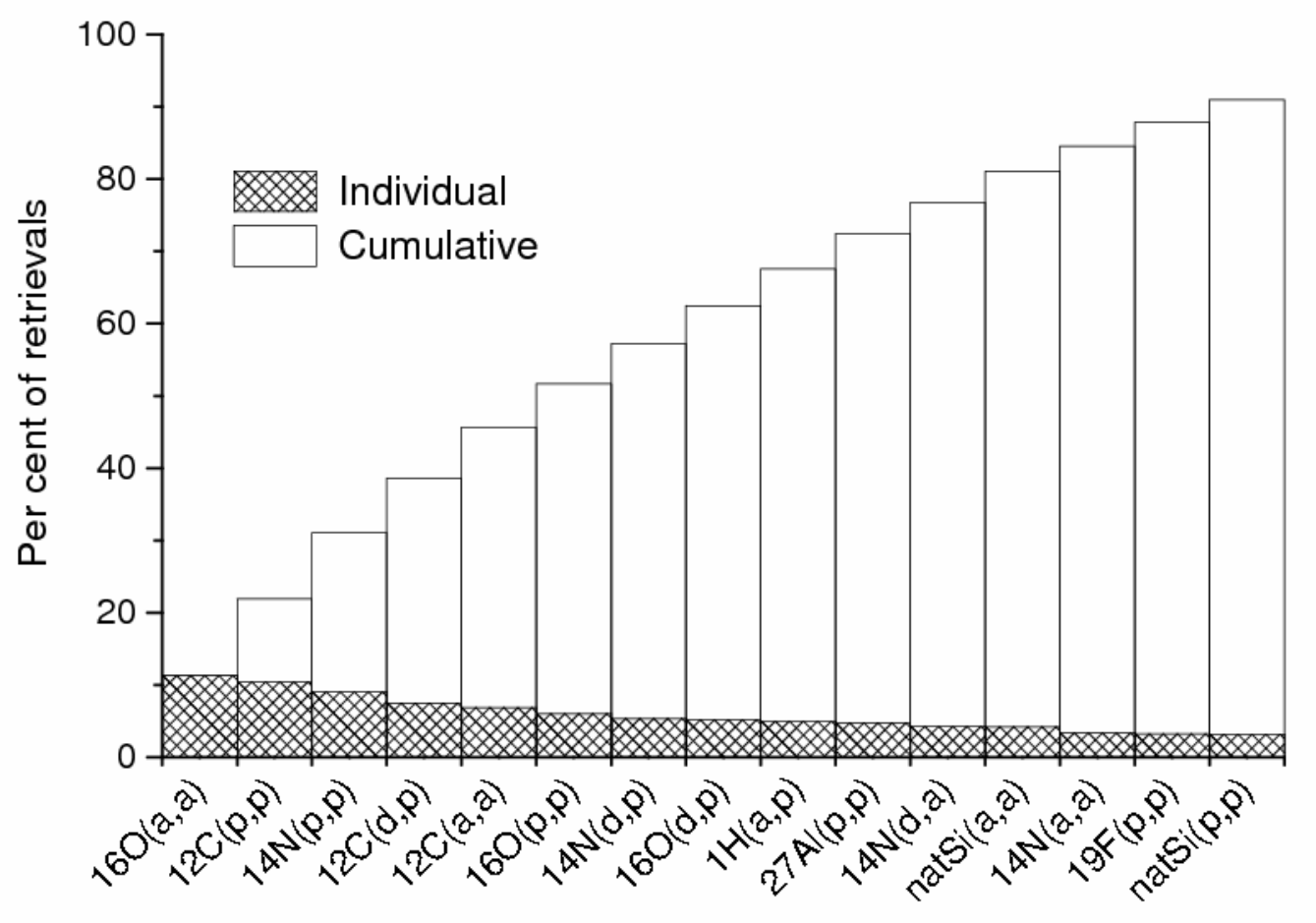

Fig.3. The IBANDL statistics showing most popular retrievals 